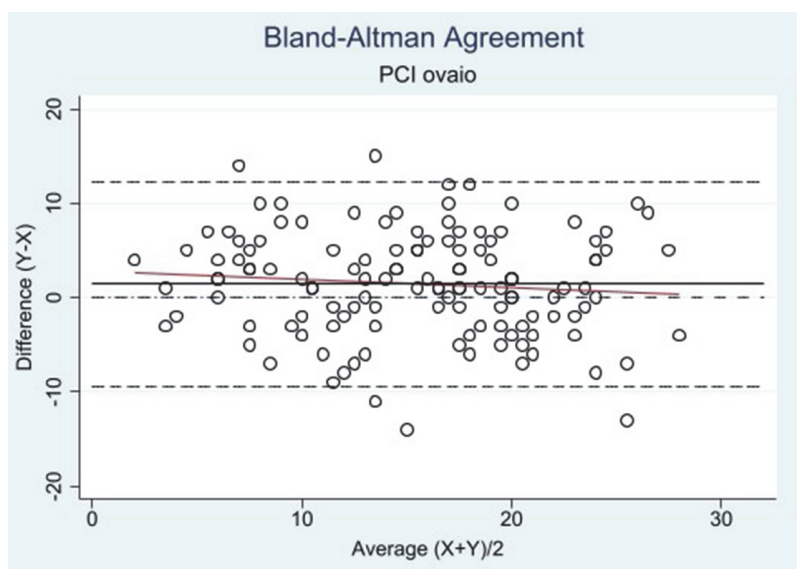

Abstract 122 Figure 1

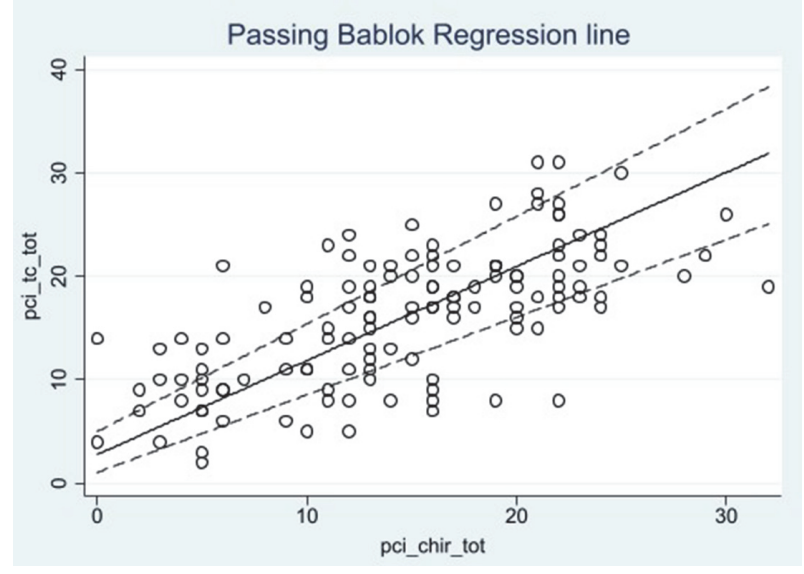

Abstract 122 Figure 2

Conclusion* CT is moderately accurate in predicting the sPCI in AOC patients. The rPCI scoring seems to be helpful and should be part of the decision-making process on surgical cytoreduction.

\section{REAL WORLD DATA OF TREATMENT AND OUTCOME OF PATIENTS WITH HIGH GRADE AOC (ADVANCED OVARIAN CANCER) IN GERMANY (QS OVAR)}

${ }^{1} \mathrm{P}$ Harter ${ }^{*},{ }^{1} \mathrm{~A}$ Du Bois, ${ }^{2} \mathrm{~F}$ Hilpert, ${ }^{3} \mathrm{M}$ Kerkmann, ${ }^{4}$ J Sehouli, ${ }^{5} \mathrm{~S}$ Mahner, ${ }^{6} \mathrm{~N}$ De Gregorio, ${ }^{7} \mathrm{~L}$ Hanker, ${ }^{1} \mathrm{~F}$ Heitz, ${ }^{8} \mathrm{~F}$ Marmé, ${ }^{2,9} \mathrm{~L}$ Wölber, ${ }^{3} \mathrm{~L}$ Holtmann, ${ }^{10} \mathrm{j}$ Pfisterer. ${ }^{1}$ Ev. Kliniken EssenMitte, Gynecology and Gynecologic Oncology, Essen, Germany; ${ }^{2}$ Jerusalem Krankenhaus, Onkologisches Therapiezentrum, Hamburg, Germany; ${ }^{3} \mathrm{MMF}$ GmbH, Dortmund, Germany; ${ }^{4}$ Charité , Klinik für Gynäkologie mit Zentrum für onkologische Chirurgie, Berlin, Germany; ${ }^{5}$ LMU University Hospital, Obstetrics and Gynecology, Munich, Germany; ${ }^{6}$ SLK Kliniken, Frauenklinik, Heilbronn, Germany; ' Universitätsklinikum Schleswig-Holstein, Campus Lübeck, , Gynecology and Obstetrics, Lübeck, Germany; ${ }^{8}$ Medical Faculty Mannheim, Heidelberg University, Frauenklinik, Mannheim, Germany; ${ }^{9}$ University Medical Center Hamburg-Eppendorf, Gynecology, Hamburg, Germany; ${ }^{10}$ Zentrum für Gynäkologische Onkologie, Kiel, Germany

\subsection{6/ijgc-2021-ESG0.342}

Introduction/Background* Recent and detailed data regarding treatment quality and outcome of patients with high grade AOC on a nationwide basis are largely unknown in Germany. Methodology All German hospitals treating patients with ovarian cancer were asked to document all patients with first diagnosis in the third quarter in 2012 and 2016. Details of tumor, treatment and outcome were documented. This analysis is focusing on patients with AOC FIGO III/IV and the endpoint PFS (progression free survival) (OS data immature for 2016 cohort). As response rates were not documented, we defined a subcohort of patients without progressive disease within 3 months after end of chemotherapy as potential candidates for an additional maintenance therapy.

Result(s)* In total, 1010 patients with high grade AOC with a median age of 65 years were documented. 774/1010 (76.6\%) were diagnosed with FIGO III disease and 947/1010 (93.8\%) had high-grade serous histologic subtype. 915/1010 (90.6\%) had primary cytoreductive surgery. Complete resection was achieved in 434/1010 (43.0\%) at primary surgery and in 54/ $1010(5.3 \%)$ at interval debulking surgery. Carboplatin/paclitaxel/bevacizumab (TCB) was the primary systemic therapy in $627 / 1010(62.1 \%)$ and carboplatin/paclitaxel (TC) in 292/1010 (28.9\%). Median PFS in patients with primary surgery and complete resection was 29.7 months (95\% CI 27.4-34.2) compared to 17.2 months $(95 \%$ CI 15.7-18.9) in all other patients $(\mathrm{p}<0.001$ : HR 0.49 , 95\% CI 0.42-0.57). Median PFS in patients with TCB was 23.3 months (95\% CI 22.0-25.3) and 18.5 months (95\% CI 15.7-21.9) in patients treated with TC $(p=0,083$ : HR $0.87,95 \%$ CI 0.74-1.02). In total, 899/ 1010 (89\%) did not progress within 3 months.

Conclusion* Most patients in Germany with high grade AOC are treated with primary surgery followed by carboplatin/paclitaxel/bevacizumab. Depending on biomarker status most patients are potential candidates for a PARP-inhibitor maintenance therapy.

\section{COULD CHANGE IN NEOADJUVANT CHEMOTHERAPY REGIMEN AFTER 3 CYCLES LEAD TO BETTER OUTCOMES IN INOPERABLE OVARIAN CANCER?}

'S Adeleke*, 'N Brown, 'A Choy, 'Z Ahmed, 'A Tsirekidze, 'V Ramachandran, ${ }^{1} S$ Ramanaidu, 'A Haslam, 'J Galante, ${ }^{2} \mathrm{~S}$ Shi, 'S George, ${ }^{1} \mathrm{~A}$ Davis, ${ }^{1} \mathrm{C}$ Mikropoulos, 1J Waters, 'J Summers, 'R Jyothirmayi. 'Maidstone Hospital, United Kingdom; ' $G u y$ 's Hospital, United Kingdom

\subsection{6/ijgc-2021-ESG0.343}

Introduction/Background* Multiple studies have assessed the timing of surgery in high grade serous ovarian cancer (HGSOC). There is some evidence that neoadjuvant chemotherapy (NACT) followed by interval debulking surgery is non-inferior to primary cytoreductive surgery. The ICON $8 b$ study results are awaited to evaluate the use of upfront bevacizumab in these patients. However, there is currently limited evidence supporting switching chemotherapy regimens after 3 cycles of NACT when disease remains inoperable. In this retrospective study, we evaluated survival outcomes following a change of chemotherapy regimen in previously inoperable patients.

Methodology Patients with HGSOC treated with NACT from 2015 to 2020 were identified from 4 NHS trusts across Kent, UK. Chemotherapy regimens, number of cycles, debulking status/residual disease were extracted from electronic medical records. Kaplan-Meier analyses and Logrank tests were used to assess survival outcomes.

Result(s)* 149 patients were identified. 59\% (88/149) were deemed operable after 3 cycles of NACT (carboplatin + paclitaxel 3 weekly), with $39 \%(34 / 88)$ of these achieving optimal debulking. $41 \%(61 / 149)$ were inoperable after three cycles of NACT, with $79 \%(48 / 61)$ of these remaining inoperable after 